Sessions d'étude - Société canadienne d'histoire de l'Église catholique

\title{
Les intendants de la Nouvelle-France et la religion
}

\section{Jean-Claude Dubé}

Volume 45, 1978

URI : https://id.erudit.org/iderudit/1007121ar

DOI : https://doi.org/10.7202/1007121ar

Aller au sommaire du numéro

Éditeur(s)

Les Éditions Historia Ecclesiæ Catholicæ Canadensis Inc.

ISSN

0318-6172 (imprimé)

1927-7067 (numérique)

Découvrir la revue

Citer cet article

Dubé, J.-C. (1978). Les intendants de la Nouvelle-France et la religion. Sessions d'étude - Société canadienne d'histoire de l'Église catholique, 45, 5-17.

https://doi.org/10.7202/1007121ar

Tous droits réservés @ Les Éditions Historia Ecclesiæ Catholicæ Canadensis Inc., 1978
Ce document est protégé par la loi sur le droit d'auteur. L'utilisation des services d’Érudit (y compris la reproduction) est assujettie à sa politique d'utilisation que vous pouvez consulter en ligne.

https://apropos.erudit.org/fr/usagers/politique-dutilisation/ 


\section{Les intendants de la Nouvelle-France et la religion}

Quinze personnes furent nommées par le roi à l'intendance de Québec entre 1663 et 1760 . Mes recherches ont porté depuis quelques années sur l'analyse des diverses composantes sociales de ce groupe. J'ai tenté au fond de répondre à la question suivante : quelle sorte d'hommes le roi choisissait-il pour administrer le Canada?

Une première réponse, que je retiendrai ici, concerne l'origine sociale (ou socio-professionnelle); il s'agit d'un milieu homogène, où dominent la magistrature et les finances. En voici un exemple précis : les professions des pères des intendants : 10 ont servi comme magistrats ( 5 magistrats de province, 2 de la haute magistrature un intendant de province et un conseiller au Parlement de Paris -2 intendants de marine et un procureur au Parlement). Pour la finance, 7 cas : 5 sont officiers de finance et 2, financiers - ils furent tous les deux taxés par la Chambre de justice de 1661, l'un pour 800,000 livres, l'autre pour 150,000 . Une seule exception : le père de Claude-Thomas Dupuy, qui est marchand bourgeois de Paris. Ce statut social commande la richesse : les fortunes des pères oscillent entre 40,000 et 820,000 livres.

Une deuxième réponse à ma question initiale concerne ce qu'on pourrait appeler le comportement culturel ${ }^{1}$. Il est normal dans ce milieu d'aller au collège et de fréquenter ensuite la Faculté de Droit. L'intérêt pour les choses de l'esprit transparaît dans les bibliothèques ou la décoration des demeures; il y a des degrés, bien sûr, mais il n'est absent nulle part. De plus nous jugeons remarquable la contribution des intendants de Québec à la vie scientifique de leur époque : 4 des 15 , c'est à dire plus du quart, y participèrent activement. Bouteroue, un des plus grands, sinon le plus brillant numismate français de son temps; Dupuy, aux connaissances uni-

1 Voir J.C. DubÉ, «Les intendants de la Nouvelle-France et la République des Lettres», dans RHAF, v. 29 (1975), pp. 31-48. 
verselles et dont les recherches en physique intéressèrent l'Académie des Sciences; Antoine-Denis Raudot, très versé en géographie et en ethnographie - nous ne connaissons malheureusement pas le catalogue de sa riche bibliothèque (2000 volumes) - qui anima, avec le comte d'Estrées le cercle de lettrés qui, au début du XVIII siècle, dirigea le ministère de la marine. J'ai aussi retrouvé à la Bibliothèque nationale quelques lettres échangées entre François de Beauharnois et Dom Montfaucon, le célèbre érudit bénédictin, qui fut l'un des fondateurs de l'ar:héologie française.

Ce que je voudrais maintenant aborder, c'est leur "religion», pour reprendre. l'expression d'Henri Busson dans son livre La religion des Classiques ${ }^{2}$. Voilà certes un domaine relativement délicat et insaisissable, non seulement parce qu'il s'agit d'une période où la pratique est universelle, mais encore parce que ce sont des personnages officiels - la correspondance officielle donne naturellement peu d'indications sur leurs idées religieuses, et dans les épaves qui surnagent de la correspondance privée nous n'avons rien trouvé de significatif ${ }^{3}$. Le comportement religieux est commandé par leur position : assistance aux actes rituels, par exemple, ou charité envers les pauvres ou les communautés. Quant au comportement moral, il nous échappe en grande partie ; on peut toujours parler des galanteries de Louis Robert, le premier intendant nommé, mais qui ne vint pas (je soupçonne Laval qui était alors à Paris d'avoir protesté contre cette nomination - d'ailleurs l'oncle de ce Robert, chanoine de Notre-Dame de Paris avait eu lui aussi des enfants naturels, scandale sans doute pour le sévère Laval), et de celles de Bigot. Mais au delà de ces détails nous ne connaissons pratiquement rien de leur «morale».

L'on a beaucoup parlé de l'enrichissement scandaleux de Bigot et de ses amis. On leur fit de 1761 à 1763 un procès retentissant. Bigot fut d'abord condamné à mort, puis au bannissement à vie et à la confiscation de ses biens ${ }^{4}$. Mais il protesta toujours de son innocence, parce qu'il considérait que ces pratiques courantes dans les milieux politiques étaient pour ainsi dire normales - Colbert,

2 Paris, P.U.F., 1948.

3 Voir la correspondance de Michel VI BÉGon dans le Fonds Bégon, à Archives de l'Université de Montréal.

4 Voir G. FréGault, François Bigot, administrateur français, Montréal, Institut d'Histoire de l'Amérique Française, 1948, 2 v. 
Richelieu, Mazarin ${ }^{5}$ le plus extraordinaire de tous à cet égard, n'étaient-ils pas morts fort riches ? - il n'y avait pas de raison de les lui reprocher plus qu'aux autres. Morale sans doute assez ambiguë, et qu'entretenait la confusion qui subsista pendant tout l'ancien régime entre affaires privées et affaires publiques ${ }^{6}$.

Il serait hasardeux d'aller plus avant dans cette direction. Mon propos sera plus modeste. Tenant compte des sources disponibles, je voudrais traiter de deux points sur lesquels il est possible d'avoir des données concrètes. Il y a d'abord moyen de définir la place de ces 14 familles dans l'Église, ce qui permettra de caractériser en quelque sorte le milieu dans lequel ont été éduqués les intendants, ou dans lequel ils ont vécu une partie de leur vie. Nous tenterons ensuite à partir d'un document intéressant, le testament, de découvrir certains aspects de leur mentalité religieuse.

C'est au moyen des généalogies que j'ai pu retracer les vocations ecclésiastiques et religieuses ${ }^{7}$. J'ai aussi tenu compte des familles alliées, celles de la femme ou de la mère de l'intendant, par exemple. Ainsi les Bouteroue n'ont, à ma connaissance, aucune vocation dans leurs rangs, mais Marie Lescot, épouse de Claude, l'intendant, a un frère prêtre et une sœur religieuse. Il va sans dire que ces données sont minimales, parce que dans la plupart des cas les reconstitutions sont incomplètes à cause de la pauvreté de la documentation. Ainsi il n'est pas sûr que les notaires Bouteroue de Meaux n'aient pas compté un parent dans le clergé au XVI ${ }^{\mathbf{e}}$ siècle, mais nous ne pouvons le savoir pour le moment.

Le tableau I regroupe les résultats de mon enquête. J'ai distingué les $\mathrm{XVI}^{\mathrm{e}}, \mathrm{XVII}^{\mathrm{e}}$ et $\mathrm{XVIII}^{\mathrm{e}}$ siècles, en $\mathrm{y}$ relevant les prêtres, les religieux, les évêques et les religieuses. J'ai ajouté pour certaines familles des détails qui semblaient importants.

Voici quelques-unes des remarques que suggère le tableau. Pas une famille qui, pour l'époque qui nous intéresse n'ait au moins deux personnes engagées dans l'Église. Ce qui est encore plus révélateur, à notre sens, mais que nous ne pouvions facilement

5 Voir D. Dessert, "Pouvoir et finance au XVIIe siècle : la fortune du Cardinal Mazarin », dans Revue d'Histoire moderne, t. 23 (1976), p. 161ss.

6 Voir J. BosHeR, French Government and Society 1500-1850, London, Athlone Press, 1973, p. 19ss et J.C. DuBÉ, infra note 9.

7 Le livre que je prépare sur les intendants comprendra en appendice les généalogies de tous les intendants. 
Tableau I

\section{VOCATIONS ECCLÉSIASTIQUES ET RELIGIEUSES DANS LES FAMILLES}

DES INTENDANTS DE LA NOUVELLE-FRANCE

\begin{tabular}{|c|c|c|c|c|c|c|c|c|c|c|c|c|c|c|}
\hline \multirow[b]{2}{*}{ Familles } & \multicolumn{4}{|c|}{ XVI• siècle } & \multicolumn{4}{|c|}{ XVII siècle } & \multicolumn{4}{|c|}{ XVIIIe siècle } & \multirow{2}{*}{ Total } & \multirow{2}{*}{$\begin{array}{l}\text { Particularités } \\
\text { SIGLES : } \\
\text { P: Prêtre; Rx: Religieux; Ev.: Evêque; Rse: Religieuse }\end{array}$} \\
\hline & $\mathbf{P}$ & $\mathbf{R x}$ & Ev. & Rse & $\mathbf{P}$ & $\mathbf{R x}$ & Ev. & Rse & $\mathbf{P}$ & $\mathbf{R x}$ & Ev. & Rse & & \\
\hline Robert (Louis) & 2 & & & & 4 & & & 5 & 1 & & & 1 & 13 & \\
\hline Talon - Paris & 1 & 1 & & & 4 & & * & 1 & & & & & 13 & * Charles Talon, nommé év. de Léon, décline cet honneur. \\
\hline - Chalons & & 1 & & & 4 & & & 1 & & & & & & \\
\hline Bouteroue & & & & & 1 & & & 1 & & & & & 2 & $\begin{array}{l}\text { Il s'agit du frère et de la sceur de Marie Lescot, femme } \\
\text { de Claude Bouteroue. }\end{array}$ \\
\hline Duchesneau & 1 & & & & & 1 & & 1 & & & & & 3 & Alliés aux Tronson. \\
\hline De Meulles & & & $*$ & & & & & 2 & & & & & 2 & $\begin{array}{l}\text { * Elisabeth Briçonnet, mère de Jacques De Meulles, est de } \\
\text { la célébre famille qui au XVI॰ s.: } 6 \text { év. et un cardinal. }\end{array}$ \\
\hline Bochart & & & 1 & & $4^{*}$ & 2 & 2 & 3 & 3 & & & 3 & 18 & $\begin{array}{l}\text { * René Bochart et son fils, Samuel, sont pasteurs protes- } \\
\text { tants à Caen. Apparentés au Cardinal de Richelieu et à } \\
\text { Louis Tronson. }\end{array}$ \\
\hline Beauharnois & 2 & & & 1 & 5 & & & 3 & & & & 1 & 12 & $\begin{array}{l}\text { Au XVIIe s., une sainte, Madame de Miramion, et un } \\
\text { abbe perverti, F. T. de Choisy. }\end{array}$ \\
\hline Raudot & & & & & & & & 2 & & & & 1 & 3 & \\
\hline Bégon & & & & & 1 & & & 6 & & & 1 & 2 & 10 & \\
\hline Robert (E.N.) & & & & & 1 & & & & 1 & & & & 2 & \\
\hline Chazel & & & & & 3 & & & & 1 & & & 1 & 5 & \\
\hline Dupuy & & & & & 3 & & & & 2 & 1 & & 3 & 9 & \\
\hline Hocquart & & & & & 1 & & & & 1 & & & 1 & 3 & \\
\hline Bigot & & & & & $\mathbf{1}$ & & & 2 & & 1 & & 2 & 6 & Apparentés aux Bonneau (Madame de Miramion). \\
\hline
\end{tabular}


inscrire, c'est que la majorité des intendants ont au moins un frère prêtre ou une sceur religieuse; ceux qui échappent à cette règle sont Claude-Thomas Dupuy, Henry de Chazel, Edme-Nicolas Robert et les deux Raudot ; or Dupuy a trois oncles prêtres, Chazel, deux, Robert un au moins; quant aux Raudot ils sont apparentés aux Talon.

Certaines familles sont mieux représentées que les autres dans l'Église, comme on peut le constater. Les Talon par exemple, qui comptent 8 prêtres au $\mathrm{XVII}^{\mathrm{e}}$ siècle, dont trois dans la seule famille de l'intendant. Charles Talon, le fils d'Omer, le premier des célèbres avocats parisiens, fut nommé à l'évêché de Léon, mais déclina cet honneur. Le célèbre abbé Pucelle, conseiller clerc au Parlement de Paris, et janséniste influent du temps de la Régence, était aussi leur cousin.

Chez les Bégon par contre, c'est la part importante des femmes qui surprend ; en fait c'était devenu comme une tradition pour elles d'entrer au couvent. Une sœur de Michel IV, carmélite, meurt en odeur de sainteté en $1673 ; 3$ autres étaient visitandines. Deux générations plus tard, encore trois religieuses, puis une autre au milieu du XVIII ${ }^{\mathrm{e}}$ siècle : la propre fille de l'intendant. Le frère de celui-ci fera une belle carrière dans l'épiscopat.

Pour les Robert (Louis) la tradition est encore plus longue, elle se prolonge sur trois siècles. Déjà au $\mathrm{XVI}^{\mathrm{e}}$ siècle les juristes d'Orléans avaient des frères chanoines. Louis lui-même comptait deux frères prêtres et une sœur religieuse au Calvaire de Paris. Sa fille Louise se mariera en 1706 au petit-fils du Duc de Beauvilliers, célèbre dévot du temps de Louis XIV; de ses 5 enfants, un sera Abbé de Livry, l'autre chevalier de Malte, une fille se fera religieuse bénédictine à Mortagne.

Les Bochart surclassent toutes les autres familles par l'importance numérique et la qualité de leur engagement au service de l'Église. Henri Brémond n'a pas manqué de le noter dans son monumental ouvrage sur l'histoire du sentiment religieux en France ${ }^{8}$.

En fait ils avaient avec la hiérarchie des liens étroits, dès le $\mathrm{XVI}^{\mathrm{e}}$ siècle. Jean II avait en effet épousé Jeanne Simon, dont l'oncle Jean Simon, évêque de Paris, lui donna en dot la terre de

8 H. BRÉMOND, Histoire littéraire du sentiment religieux en France, Paris, 1921, t. II passim. 
Champigny, seigneurie qui leur appartiendra jusqu'à la Révolution. Jean IV, lui, avait épousé une parente de l'évêque de Meaux, Guillaume Briçonnet; il fut pour la jeune Madame Acarie, qui à cause des Luillier était parente de ses cousins, les Bochart de Ménillet, une sorte de conseiller spirituel. Rien d'étonnant alors si la famille Bochart se trouve au XVII ${ }^{e}$ siècle intimement mêlée au mouvement de la Contre-Réforme. Le capucin, Charles Bochart, connu sous le nom de "Père Honoré », qui fut en rapport étroit lui aussi avec Madame Acarie, jouissait d'une grande réputation de sainteté : sa cause de béatification a été en effet introduite à Rome; sa nièce Lyée Bochart entre, après la mort de son mari, à l'Abbaye de Port-Royal. Louis Tronson, le grand spirituel français de la fin du XVII ${ }^{e}$ siècle était leur cousin. Par ailleurs il ne faudrait pas oublier que le Cardinal de Richelieu comptait Antoine Bochart parmi ses arrière-grands-pères, et se trouvait cousin au $4^{\mathrm{e}}$ degré de Jean $\mathrm{V}$ qu'il nomma surintendant des finances.

Il est donc normal, compte tenu de ce que nous venons de relater, de retrouver à cette époque même les Bochart bien représentés dans l'épiscopat français. Guillaume Bochart, l'un des 3 frères de Jean VIII (notre intendant) qui entrèrent dans les ordres, fut nommé en 1687 évêque de Valence; son cousin, François Bochart de Saron, avait été choisi quelques mois auparavant pour le siège de Clermont. Dans ces circonstances, ce n'est pas une coïncidence que dans l'espace de quelques mois, en 1686 et 1687 , se soient faites dans cette famille trois importantes nominations: les deux que nous venons de mentionner, et celle de Jean VIII à Québec, Cette dernière est un exemple fort typique de patronage politico-religieux, si j'ose dire, puisqu'elle est due à la pression de Louis Tronson, alors supérieur de Saint-Sulpice, et du Duc de Beauvilliers, gendre de Colbert sur Seignelay, fils du même Colbert et ministre de la marine. Tronson était, je crois, directeur spirituel de Beauvilliers, et Bochart était mêlé de quelque façon à ce milieu dévot 9 .

Notons enfin que jusqu'à la Révolution au moins la descendance de Jean VIII fut représentée dans le clergé : son fils Louis-Alphonse, chanoine de la Sainte Chapelle, et le neveu de

9 Voir G. Lizerand, Le Duc de Beauvilliers 1648-1714, Paris, 1933, et J.C. DubÉ, "Colbert et l'intendance de Québec", dans Revue de l'Université d'Ottawa, 1977, p. 292ss. 
celui-ci, Jean IX, mort en 1791, vicaire général du diocèse de Macon.

Jacques De Meulles, le seul des intendants qui appartînt à un ordre de chevalerie religieuse, celui de Saint-Lazare et NotreDame du Mont Carmel, descendait par sa mère de la célèbre famille Briçonnet, qui au XVI ${ }^{\text {e }}$ siècle avait donné au Royaume de brillants administrateurs, spécialistes des finances, et d'éminents ecclésiastiques, dont un cardinal et six évêques; le plus célèbre desquels est certes Guillaume, l'évêque de Meaux, qui protégea Lefebvre d'Étaples et fut l'âme de la pré-réforme française.

Notre tableau donne aussi d'intéressantes indications sur les processus de promotion sociale. L'accès à la magistrature ou à la bonne bourgeoisie s'accompagne ordinairement de vocations. Les familles des intendants du XVIII ${ }^{e}$ siècle - sauf le premier, Beauharnois, nommé en 1702 - n'avaient pas comporté d'ecclésiastiques au XVI ${ }^{e}$ siècle. Les Raudot sont de petits marchands d'Arnayle-Duc, les Chazel, paysans dans le Gard, les Dupuy, humbles marchands ou artisans à Ambert en Auvergne ; il en va semblablement des autres. L'entrée dans le clergé se fera au XVII ${ }^{\text {e siècle. }}$ Pour tous ceux qui furent nommés au XVII ${ }^{\mathrm{e}}$ siècle, plus précisément de 1663 à 1702 - sauf Bouteroue - les vocations avaient marqué dès le siècle précédent la montée dans l'échelle sociale. Les Robert, tout comme les Beauharnois, avaient placé leurs membres dans le chapitre d'Orléans, les Duchesneau, dans celui de Tours. L'évêque de Paris était l'oncle de Jeanne Simon, épouse de Jean II Bochart. Le succès des Talon à Paris et à Châlons avait été précédé avant 1600 par trois vocations ecclésiastiques.

L'Église est donc présente dans toutes ces familles et dans plus d'un tiers d'entre elles de façon spectaculaire : plusieurs évêques, plusieurs personnes remarquables par la sainteté de leur vie. Peu d'influences jansénistes, mais par contre une active participation aux divers courants ascétiques et mystiques qui marquèrent la Contre-Réforme française - le Père Honoré par exemple au début du siècle, ou Madame de Miramion à la fin. Ceci n'exclut pas, on l'aura remarqué les dimensions sociologiques ou économiques du phénomène religieux : influences familiales ou politiques dans les nominations ou même dans les « vocations».

Les intendants ont donc généralement eu toutes les chances de par leur éducation première et de par celle qui leur a été incul- 
quée au collège, de recevoir une formation imprégnée du christianisme le plus authentique.

Pour connaître comment ils répondirent aux incitations de ce milieu, il nous faudrait maintenant étudier leurs comportements. Nous avons pour le faire bien peu de moyens. Nous disposons toutefois d'un document qui peut donner des indications, le testament.

Nous en avons retrouvé 7 sur 15 . Ce ne sont pas de longs documents; ils contiennent (avec les codicilles) de 4 à 500 mots ; une seule exception : celui de Jean Talon, qui fait près de 3000 mots ${ }^{10} .4$ des 7 sont olographes; c'est le cas de ceux qui se disent en santé. En fait un seul, François de Beauharnois, teste à l'article de la mort. Deux seulement ont des héritiers directs, les autres sont célibataires ou n'ont pas d'enfants.

Pour étudier le contenu de cet important document j'ai aussi fabriqué un tableau. Les intendants y figurent en ordre chronologique, suivant la date du testament, non la date de la nomination à Québec ; en horizontal j'ai regroupé dans 10 articles, les divers éléments qu'on retrouve dans le document, suivant plus ou moins les analyses de Pierre Chaunu dans son récent livre sur la mort à Paris sous l'Ancien Régime ${ }^{11}$.

(I-II) Quand le notaire se présentait devant son client, il avait quelques formules à lui proposer ; selon Chaunu le choix que faisait alors le testateur est significatif. La formule de Louis Robert est ainsi toute classique :

Premièrement il recommande son âme à Dieu supliant sa divine bonté le recevoir en Paradis et luy pardonner ses peschez par les merites infinis du sang precieux de Jesus Christ repandu

10 Le testament de Talon que T. CHAPAIS met en appendice à son Jean Talon, intendant de la Nouvelle-France est rempli de fautes de transcription. Voir la minute au Minutier Central (MC) de Paris, Étude LVIII, 24 nov. 1694. Celui de Louis Robert, MC, ét. CXIII, 217, 25 février 1706. Celui de Jacques Raudot, MC, ét. LXVII, 404, 28 avril 1726. Celui d'Antoine-Denis Raudot, Arch. Dept. Versailles, Notaires, 29 juillet 1737, dépôt. Celui de François de Beauharnois, Arch. Dept. Loiret (Orléans), 3E 10,522. Celui de Bigot, déposé à Arch. Dept. Gironde (Bordeaux), 20 février 1778, 3E 15,028. Celui de G. Hocquart, MC, ét. II, 711, 16 avril 1783.

11 P. Chaunu, La mort à Paris, 16e $, 17^{e}, 18^{e}$ siècles, Paris, Fayard, 1978. Voir aussi M. Vovelle, Piété baroque et déchristianisation en Provence au XVIII siècle, Paris, Plon, 1973. 


\section{Tableau II}

TESTAMENTS DES INTENDANTS DE LA NOUVELLE-FRANCE

\begin{tabular}{|c|c|c|c|c|c|}
\hline $\begin{array}{l}\text { Nom } \\
\text { Date } \\
\text { Délai } \\
\text { Postérité }\end{array}$ & I. Invocation & $\begin{array}{l}\text { II. Sentiment } \\
\text { chrétien (plus } \\
\text { loin dans teste) }\end{array}$ & III. Corps & $\begin{array}{l}\text { IV. Enterrement } \\
\text { et sépulture }\end{array}$ & V. Messes \\
\hline $\begin{array}{l}\text { Talon J. } \\
1694 \\
7 \text { mois } \\
\text { s.p. } \\
\text { T. olog. }\end{array}$ & $\begin{array}{l}\text { Signe de croix, belle formule trinitaire. Prévenir } \\
\text { mort. Précieux Sang du Christ lave crimes. } \\
\text { Vierge; saints et saintes du Paradis. }\end{array}$ & $\begin{array}{l}\text { Juste colère de Dieu } \\
\text { sur ses péchés. A } \\
\text { fondé oratoire pour } \\
\text { nobles. Se preoccu- } \\
\text { pe vocation religieu- } \\
\text { se de ses nièces. }\end{array}$ & $\begin{array}{l}\text { Cour mis dans boîte } \\
\text { plomb } 24 \text { hrs après } \\
\text { décès. } \\
\text { Cour et corps inh. } \\
\text { avec cx de parents } \\
\text { Eglise N.D. Châlons. }\end{array}$ & $\begin{array}{l}\text { Inhumation modeste } \\
\text { et sans éclat, mais } \\
\text { avec dévotion et } \\
\text { grand secours de } \\
\text { prières. }\end{array}$ & $\begin{array}{l}\text { Fondation messe } \\
\text { quotidienne et anni- } \\
\text { versaire à perpettui- } \\
\text { té rente de } 450 \mathrm{~L} \\
\text { pour ce. Messes à } \\
\text { Paris dès décès } \\
\text { 200L. }\end{array}$ \\
\hline $\begin{array}{l}\text { Robert L. } \\
1706 \\
4 \text { mois } \\
2 \text { filles }\end{array}$ & $\begin{array}{l}\text { Prévenir surprise de la mort. Recommande âme } \\
\text { à Dieu. Espère aller. au Paradis. Pardon pca } \\
\text { précieux sang de J.C. Vierge; Saints et saintes. }\end{array}$ & & & & $\begin{array}{l}100 \text { messes, église } \\
\text { des Capucins. }\end{array}$ \\
\hline $\begin{array}{l}\text { Raudot J. } \\
1726 \text { mois } \\
21 \text { mois } \\
1 \text { fils } \\
1 \text { pte-fe } \\
\end{array}$ & Aucune invocation. & $\begin{array}{l}\text { "Modestie chrétien- } \\
\text { ne » dans enterre- } \\
\text { ment. }\end{array}$ & & $\begin{array}{l}\text { A la discrétion de } \\
\text { son exécuteur et } \\
\text { même lieu que lui. }\end{array}$ & $\begin{array}{l}50 \text { messes, et prières } \\
\text { au jugement de exé- } \\
\text { cuteur. }\end{array}$ \\
\hline $\begin{array}{l}\text { Raudot } \\
\text { A.D. olog. } \\
1735 \text {. } \\
29 \text { mois } \\
\text { s.p. }\end{array}$ & $\begin{array}{l}\text { Sit nomen Domini benedictum. L'homme sage } \\
\text { se «précautionne » contre la faiblesse de l'âge. }\end{array}$ & & & $\begin{array}{l}\text { Ent. des plus sim- } \\
\text { ples, pas tentures, ni } \\
\text { sonnerie... Eglise des } \\
\text { Récollets. }\end{array}$ & $\begin{array}{l}\text { Une annuelle et ser- } \\
\text { vice au bout de l'an, } \\
600 \mathrm{~L} \text {. }\end{array}$ \\
\hline $\begin{array}{l}\text { Hocquart } \\
\text { G. } 1780 \\
3 \text { ans } \\
\text { s.p. } \\
\text { olog. }\end{array}$ & Aucune invocation. & & & & $\begin{array}{l}900 \text { messes pour lui, } \\
\text { sa femme et sa } \\
\text { bell-sr. Service an- } \\
\text { nuel pr les 3. }\end{array}$ \\
\hline
\end{tabular}


Tableau II (suite)

TESTAMENTS DES INTENDANTS DE LA NOUVELLE-FRANCE

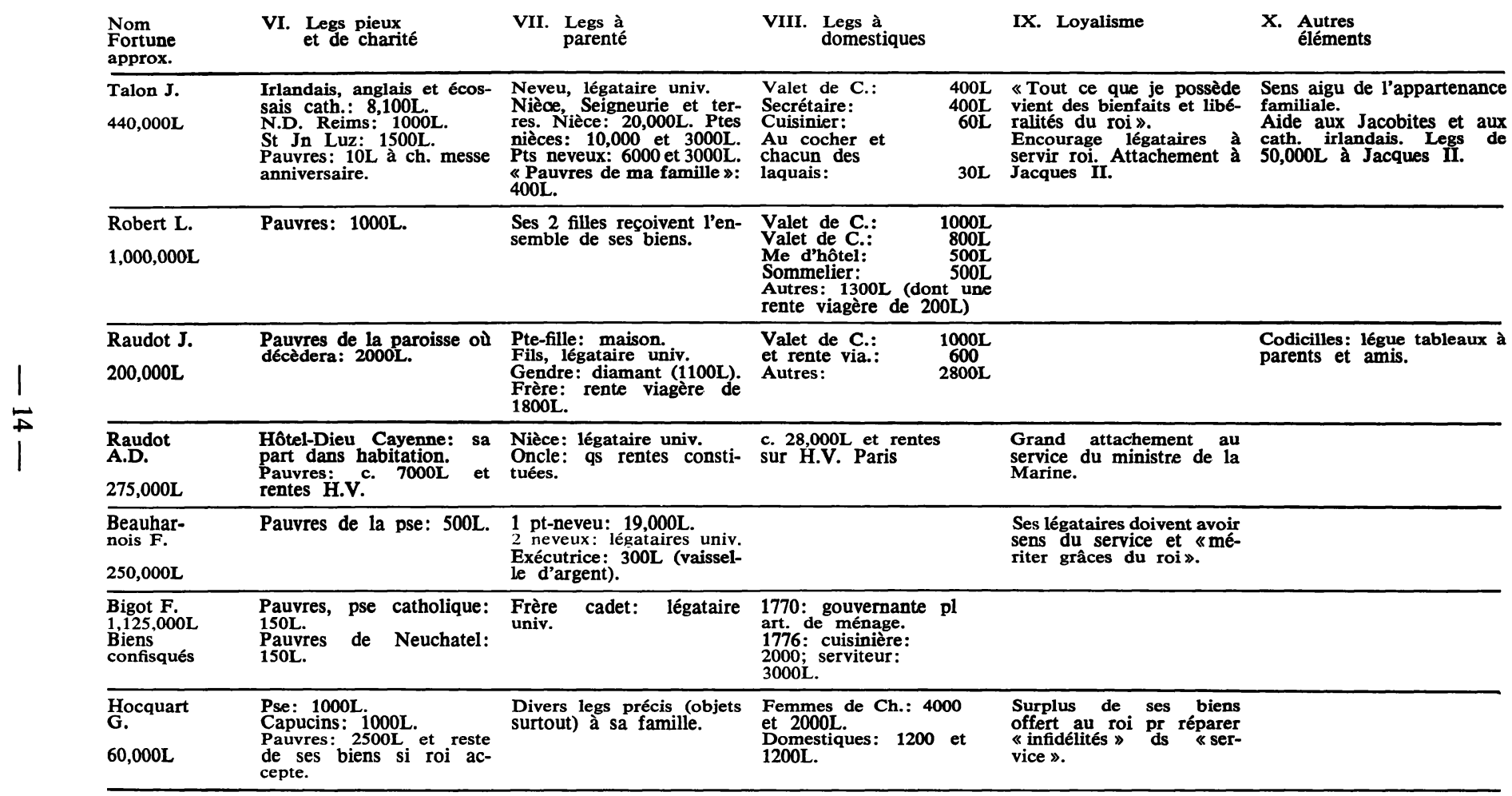


pour tous les hommes, invoquant à cet effet l'intercession de la très Sainte Vierge et de tous les Saints et Saintes.

On retrouve sensiblement les mêmes expressions chez Jean Talon, mais avec un accent bien particulier :

\begin{abstract}
Au nom du Père, du Fils et du Saint Esprit, un seul en trois personnes; Trinité sainte que j'adore, en qui je crois, en qui j'espère ... A Après avoir prié le Père Eternel de recevoir dans le sein d'Abraham mon ame purifiée de ses crimes par le sang precieux de son Fils, après avoir demandé à la Sainte Vierge ses suffrages auprès de ce même fils, aussi bien que les suffrages de tous les anges et des saints et saintes du Paradis.
\end{abstract}

Bigot, lui, ajoute une mention, qui est rare dans les testaments de l'époque : le recours à la communion des saints. Quant à AntoineDenis Raudot, il choisit un verset très connu de psaume, qui résume bien la résignation chrétienne, "Sit nomen Domini benedictum 》; c'est un choix qui me paraît original. On ne peut douter en tout cas du caractère authentiquement chrétien de ces invocations.

D'autres ont une attitude plus « moderne », leur testament ne comporte aucune invocation au début ; mais on voit par des notations subséquentes que la pensée chrétienne est loin d'être absente. Jacques Raudot demande que ses obsèques soient marquées par la « modestie chrétienne ». Beauharnois insiste sur son misérable état de pécheur et la miséricorde divine, il demande une épitaphe étonnante «Hic jacet Beauharnois peccator». Hocquart donne la majorité de ses biens aux pauvres.

(III-IV) La préoccupation du corps après la mort est absente sauf pour Beauharnois, qui exige qu'on attende 3 jours avant de le mettre en terre, et Talon, qui a prévu dans les détails ce qu'il faudra accomplir immédiatement après son décès. Quant à l'inhumation, elle sera, pour reprendre les expressions usitées ici, « modeste », « simple », «sans appareil ». Jusqu'en 1737, c'est l'enterrement dans l'église qui domine - mais nous ne savons pas où fut inhumé Louis Robert. Après cette date, c'est dans le cimetière. Notons d'ailleurs que l'ordonnance royale interdisant l'inhumation dans les églises date de 1776.

(V-VI) Le montant attribué aux messes n'est pas très élevé, si on le met en regard de leurs fortunes. Seul Talon laisse pour 
cela un capital important : 9000 livres (qui se trouve être en fait une hypothèque sur une de ses seigneuries).

Par contre les dons aux pauvres ou à des organismes religieux sont, dans tous les cas, soit aussi importants que l'argent des messes, soit supérieurs, et parfois de beaucoup. Il n'est pas possible d'évaluer exactement les sommes laissées par Antoine-Denis Raudot aux pauvres, mais elles faisaient certainement entre 10 et 15,000 livres. C'est la même chose pour Hocquart, qui veut bien leur accorder le reste de ses biens, si le roi n'accepte pas le don qu'il lui propose.

(VII-X) Les dernières dispositions ne nous intéressent pas directement ici. Beauharnois est le seul à ne rien laisser à ses domestiques. Faut-il mettre au compte d'un sens chrétien du devoir ou tout simplement d'un amour de leur vocation de fonctionnaire, l'attachement au service que l'on trouve exprimé dans 4 cas sur 7 ? Impossible de trancher. En tous cas cela prend des formes peu banales dans les cas de Talon et de Hocquart. Le premier reconnaît au roi «le droit de disposer souverainement de tout (son) bien »; le second déclare que le surplus de ses biens sera «remis au Roy par l'entremise de M. de Sartine, ministre d'État, pour qu'il en soit fait tel remploy qu'il plaira à Sa Majesté, et ce en réparation de quelques légères infidélités ... omissions et négligences par moy commises pendant le cours de mon service ». Scrupule bien rare chez un administrateur.

Il s'agit, pour tous les intendants, d'un document préparé avec soin. Il n'est que de voir la minutie avec laquelle Talon, par exemple, décrit chacune des dispositions concernant le souvenir des membres de sa famille (épitaphes, tombes, etc.) ou les legs à ses neveux et nièces. Nous croyons que les sentiments chrétiens exprimés dans le préambule ne sont pas une simple façade, mais qu'ils correspondent à quelque chose de profond chez eux - une profondeur qu'il est, bien sûr, difficile de mesurer. Un cas me paraît toutefois assez clair, celui de Talon: il est incontestable que, tout au moins à la fin de sa vie, il penchait du côté de la dévotion. Il avait en effet fondé un oratoire dans Paris «pour, dit-il, servir utilement aux personnes de qualité, qui pour faire plus utilement leurs dévotions voudront s'y retirer ». Il s'est intéressé vivement à la cause jacobite ; il lègue à cet effet beaucoup d'argent, dont 50,000 livres à Jacques II lui-même; il a été en relation à leur sujet avec Madame de Maintenon. Il se préoccupe de la vocation 
religieuse de ses petites-nièces; il pardonne généreusement à sa belle, qui lui a rendu la vie difficile en ses dernières années.

La religion des intendants? Nous la croyons donc authentique ; leur éducation avait été chrétienne, des membres de leur famille étaient d'église. Leur comportement moral avait sans doute dans certains cas été peu louable, mais ils s'en repentirent au moins sur leur lit de mort, et moururent, il y a tout lieu de croire, chrétiennement. C'est tout au moins une présomption; il est difficile, sans autre document, d'aller plus loin.

Jean-Claude DuBÉ

Université d'Ottawa 